\title{
A Uniform Asymptotic Solution for Transmitted Waves through a Plane Dielectric Interface from a Denser to a Rarer Mediums by Using Parabolic Cylinder Functions
}

\author{
Dinh Trong Quang $\cdot$ Keiji Goto $\cdot$ Toru Kawano $\cdot$ Toyohiko Ishihara
}

\begin{abstract}
When the cylindrical wave is incident on a plane dielectric interface from a denser medium to a rarer one, the asymptotic solution for the transmitted wave in the near region is different from the one in the far region. In this paper, we have derived a novel uniform asymptotic solution represented by using the parabolic cylinder function for the transmitted and scattered waves observed in the rarer medium when the cylindrical wave is incident on the plane dielectric interface from the denser medium. The validity of the uniform asymptotic solution has been confirmed by comparing with the reference solution calculated numerically. It has been clarified that the transition wave plays an important role to connect smoothly the asymptotic solution in the near region to the one in the far region through the transition region. We have shown the very interesting phenomenon that the lateral wave type transmitted wave is observed in the far and shallow region.
\end{abstract}

Key words: Parabolic Cylinder Function, Plane Dielectric Interface, Transmitted Wave, Uniform Asymptotic Solution.

\section{Introduction}

The problems of the high-frequency transmitted and scattered waves excited by a high-frequency incident wave on a plane dielectric interface consisting of two different mediums have been the important research subjects for many years in the areas of the electromagnetic theory [1] [6], the antennas and the propagation [7], [8], the optics [9] [12], the acoustics [13], [14], the metamaterials [15] [18], and so forth. The phenomena of the lateral wave [1] [6], [7], [10], [11], [13], the totally reflected ray, the evanescent wave [6], [7], [19] [23] produced by an incident wave with the incident angle greater than the critical angle of the total reflection, and the transmitted geometrical ray [19] [23] are related to the above mentioned problems.

When the cylindrical wave or the spherical wave is incident on a plane dielectric interface from a denser 1 st medium $\left(\varepsilon_{1}, \mu_{0}\right)$ to a rarer 2 nd one $\left(\varepsilon_{7}, \mu_{0}\right)$ where $\varepsilon_{1}>\varepsilon_{2}$, the observation point placed in the rarer (2nd) medium may receive only the transmitted geometrical ray in the short distance from the source and both the transmitted geometrical ray and the evanescent wave in the long distance [1], [2], [6], [9] [11], [13], [19]
[23]. Thus, the asymptotic solution in the short distance is different from the solution in the long distance. Therefore, it is necessary to derive a novel uniform asymptotic solution [6], [19] [23] which can connect smoothly two different solutions in the near region and in the far region through a transition region.

In the present study, we shall derive the novel uniform asymptotic solution for the transmitted and scattered waves observed in the rarer medium when the cylindrical wave radiated from a line source is incident on a plane dielectric interface from the side of the denser dielectric medium [6], [7], [19] [23]. We will derive the uniform asymptotic solution for the transition wave which plays an important role to connect the one solution in the near region to the other solution in the far region smoothly through the transition region [6], [7], [19] [23]. The uniform asymptotic solution may be represented by using the parabolic cylinder function [24], [25]. The validity and the applicable range of the uniform asymptotic solution are confirmed by comparing with the reference solution calculated numerically from the integral representation of the transmitted wave. We will show the very interesting phenomenon that the lateral wave type transmitted wave appears in the far and

Manuscript received August 25, 2011 ; Revised January 18, 2012 ; Accepted February 2, 2012. (ID No. 20110825-02J)

Dept. of Communication Engineering, National Defense Academy, Kanagawa, Japan.

Corresponding Author : Toyohiko Ishihara (e-mail : ishihara@cc.nda.ac.jp)

This is an Open-Access article distributed under the terms of the Creative Commons Attribution Non-Commercial License (http://creativecommons.org/licenses/ by-nc/3.0) which permits unrestricted non-commercial use, distribution, and reproduction in any medium, provided the original work is properly cited. 


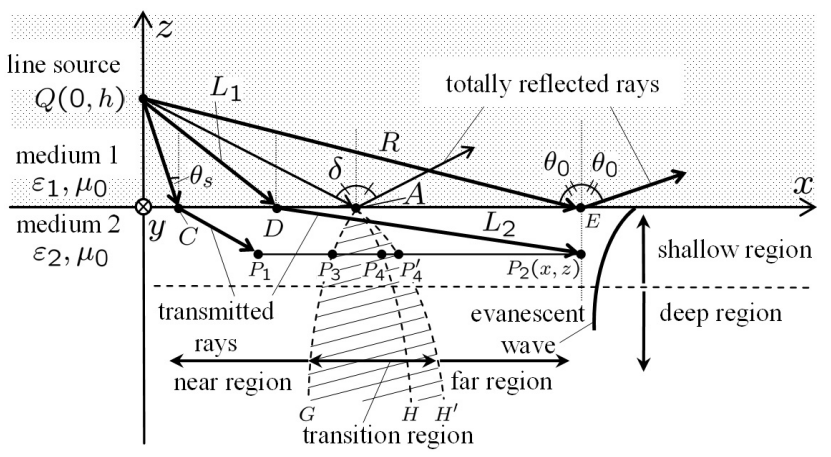

Fig. 1. Cartesian coordinate system $(x, y, z)$, plane dielectric interface at $z=0$ consisting of denser medium and rarer medium, and schematic figures for transmitted waves.

shallow region. The time convention $e^{-i \omega t}$ is adopted and suppressed here.

\section{Formulation and Integral Representations}

Fig. 1 shows $(x, y, z)$ Cartesian coordinate system, a plane dielectric interface at $z=0$ consisting of a denser medium $\left(\varepsilon_{1}, \mu_{0}\right)$ and a rarer medium $\left(\varepsilon_{2}, \mu_{0}\right)$, i.e., $\varepsilon_{1}>\varepsilon_{2}$, a electric line source $Q$ located at $(0, h)$ in the two dimensional $(x, z)$ coordinate system, totally reflected rays, transmitted rays, and an evanescent wave. The area in the rarer medium may be divided into the near region, the transition region, and the far region in the $x$-direction, and into the shallow region and the deep region in the $(-z)$-direction.

When the electromagnetic wave is radiated from the electric line source $Q(x, z)=Q(0, h)$, the electric field $E_{y}(x, z)$ observed in the rarer medium $\left(\varepsilon_{2}, \mu_{0}\right)$ may be expressed by using the Green's function $G$ as follows [6], [7], [19] [23]

$$
\begin{aligned}
& E_{y}=i \omega \mu_{0} I G, \quad G=\frac{i}{4 \pi} \int_{P_{\theta}} T(\theta) e^{i k_{1} q(\theta)} d \theta \\
& T(\theta)=\frac{2 \cos \theta}{\cos \theta+\sqrt{n^{2}-\sin ^{2} \theta}}, n=\sqrt{\varepsilon_{2} / \varepsilon_{1}} \\
& q(\theta)=R \cos \left(\theta-\theta_{0}\right)+|z| \sqrt{n^{2}-\sin ^{2} \theta}
\end{aligned}
$$

where $T(\theta)$ corresponds to the transmission coefficient while $I$ and $n$ denote, respectively, the current and the refractive index $n=\sqrt{\varepsilon_{2} / \varepsilon_{1}} . R$ and $\theta_{0}$ are defined geometrically in Fig. 1.

In Fig. 2, we have shown the original integration path $P_{\theta}$ and branch points at $\theta= \pm \delta\left(\delta=\sin ^{-1} n\right)$ associated with the branch cuts of the integrand of the Green's function $G$ in the complex $\theta$-plane. Note that the branch point at $\theta=\delta$ corresponds to the critical angle of the total reflection (see Fig. 1).

As shown in Fig. 1, only the transmitted geometrical ray is observed at $P_{1}$ in the near region. While the transmitted geometrical ray and the evanescent wave are observed at $P_{2}$ located in the far region. Therefore, it is necessary to find out the uniform asymptotic solution which can connect smoothly two different asymptotic solutions in the near region and in the far region. In the following, we shall derive from the integral (1) a novel uniform asymptotic solution applicable in the transition region [6], [19] [23] between two regions.

So far, we have considered the transverse electric (TE) case, i.e., the $y$-component of the electric field $E_{y}$ excited by the line current $I$ directing $y$-direction. When we consider the transverse magnetic (TM) case, the $y$ component of the magnetic field $H_{y}$ excited by the magnetic current $I_{m}$, directing $y$-direction and located at $Q(x, z)=Q(0, h)$ (see Fig. 1), may be obtained directly from $(1) \sim(3)$ via the duality replacements as follows;

$$
H_{y}=i \omega \varepsilon_{2} I_{m} G_{h}, \quad G_{h}=\frac{i}{4 \pi} \int_{P_{\theta}} T_{h}(\theta) e^{i k_{1} q(\theta)} d \theta
$$

where $T_{h}(\theta)$ corresponds to the transmission coefficient for the TM case and may be given by

$$
T_{h}(\theta)=\frac{2 n \cos \theta}{n^{2} \cos \theta+\sqrt{n^{2}-\sin ^{2} \theta}}, n=\sqrt{\varepsilon_{2} / \varepsilon_{1}}
$$

Note that the integrand of the Green's function $G_{h}$ in (4a) associated with (4a) and (3) possesses the branch points at the same places $\theta= \pm \delta\left(\delta=\sin ^{-1} n\right)$ with those of the integrand of the Green's function $G$ in (1) associated with (2) and (3) (see Fig. 2).

Thus, the asymptotic analysis method for the TM case represented by (4a) associated with (4b) and (3) is very

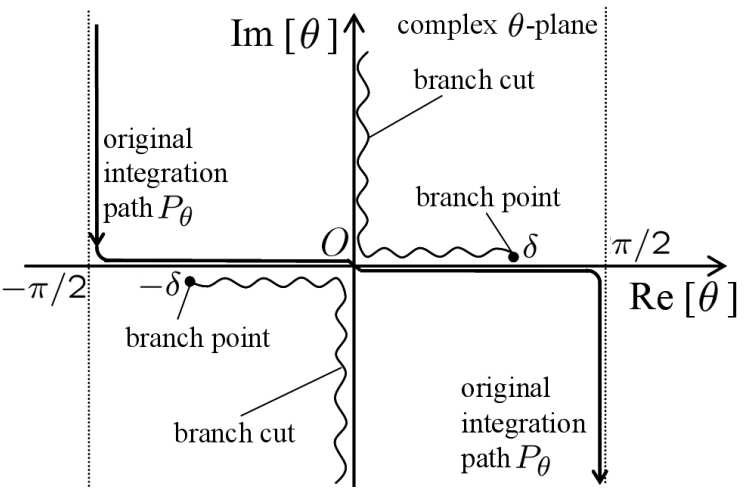

Fig. 2. Original integration path $P_{\theta}$ and branch points $\theta= \pm \delta$ associated with branch cuts in the complex $\theta$-plane. 
similar to the one for the TE case represented by (1) associated with (2) and (3). Therefore, in the following, we shall consider only the TE case represented by (1) $\sim(3)$. Note, however, that the Brewster angle $\theta_{B}$ $\left(=\tan ^{-1} n\right)$ at which the reflection coefficient of the geometrically reflected ray vanishes exists in the TM case. This phenomenon does not appear in the TE case.

\section{Asymptotic Solutions for Transmitted Waves}

\section{3-1 Asymptotic Solution in Transition Region and in Near Region}

In this section, we will derive from (1) the asymptotic solution for the transmitted wave observed in the transition region between the dotted curves $A G$ and $A H$ shown by the shaded region in Fig. 1.

In Fig. 3, we show two saddle points $\theta_{s}$ and $\theta_{e}$ obtained from the saddle point equation $(d / d \theta) q(\theta)=0$ or

$$
R \sin \left(\theta-\theta_{0}\right)+|z| \frac{\sin \theta \cos \theta}{\sqrt{n^{2}-\sin ^{2} \theta}}=0
$$

The saddle point equation in (5) can be solved analytically by obtaining first the quartic equation from (5) and then solving the quartic equation by applying the Ferrari's method [26]. Four solutions may be obtained from the quartic equation. However, one may show that only two solutions $\theta_{s}$ and $\theta_{e}$ satisfy the original saddle point equation in (5). One may also show that two solutions $\theta_{s}$ and $\theta_{e}$ obtained analytically agree excellently with the numerical solutions $\hat{\theta}_{s}$ and $\hat{\theta}_{e}$, respectively, obtained by applying the Muller's method [27].

In this case, although (5) produces two saddle points $\theta_{s}$ and $\theta_{e}$ (see Fig. 3), it can be shown that only the saddle point $\theta_{s}$ contributes to the integral (1) [6], [19] [23]. Thus, by deforming the original integration path $P_{\theta}$ into the steepest descent path $S D P_{\theta_{S}}$, one may obtain

$$
G=\frac{i}{4 \pi} \int_{S D P_{\theta_{S}}} T(\theta) e^{i k_{1} q(\theta)} d \theta
$$

Since the saddle point $\theta_{s}$ is located near the branch point at $\theta=\delta$, the asymptotic solution obtained from the isolated saddle point technique [28] produces the errors. Therefore, one may define the transmitted wave $G$ as follows.

$$
G=G_{g o}+G_{t r a n}
$$

where $G_{g o}$ denotes the transmitted geometrical ray solution obtained from the isolated saddle point technique [28] and is given by

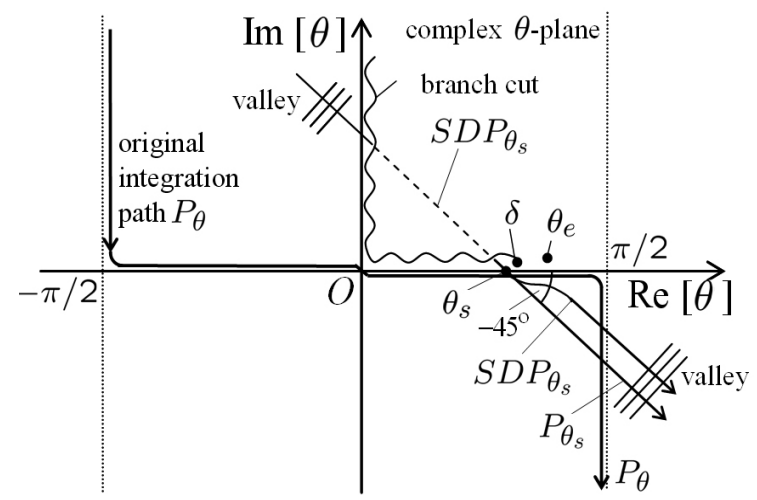

Fig. 3. Saddle points $\theta_{s}$ and $\theta_{e}$ and steepest descent path $S D P_{\theta_{S}}$ through saddle point $\theta_{S}$ in the complex $\theta$ plane. The steepest descent path $S D P_{\theta_{S}}$ and the path $P_{\theta_{S}}$ are shown by the solid lines ( $\longrightarrow$ ) when those paths run on the upper sheet and by the dashed lines $\left(--_{-}\right)$when the paths run on the lower sheet.

$$
\begin{gathered}
G_{g o}=\frac{i}{4 \pi} T\left(\theta_{s}\right) \sqrt{\frac{2 \pi}{k_{1}\left|q^{\prime \prime}\left(\theta_{s}\right)\right|}} e^{i k_{1} l_{1}+i k_{2} l_{2}-i \pi / 4} \\
q^{\prime \prime}\left(\theta_{s}\right)=-\cos \theta_{s}\left\{R \cos \theta_{0} \sec ^{2} \theta_{s}+|z| \frac{n^{2} \cos \theta_{s}}{\left(n^{2}-\sin ^{2} \theta_{s}\right)^{3 / 2}}\right\}
\end{gathered}
$$

The asymptotic solution $G_{g o}$ represents clearly the transmitted geometrical ray $Q \rightarrow C \rightarrow P_{g o}$ observed at $P_{g o}$ shown in Fig. 4. The geometrical parameters $l_{1}, l_{2}$, $R,|z|$, and $\theta_{0}$ are defined in Fig. 4.

While $G_{\text {tran }}$ in (7) denotes the transition wave which plays an important role only in the transition region between the curves $A G$ and $A H$ or in the transition region $P_{3}<x<P_{4}$ for the given depth $|z|$ (see Fig. 4) and is defined as follows.

$$
G_{\text {tran }}=G-G_{g o}
$$

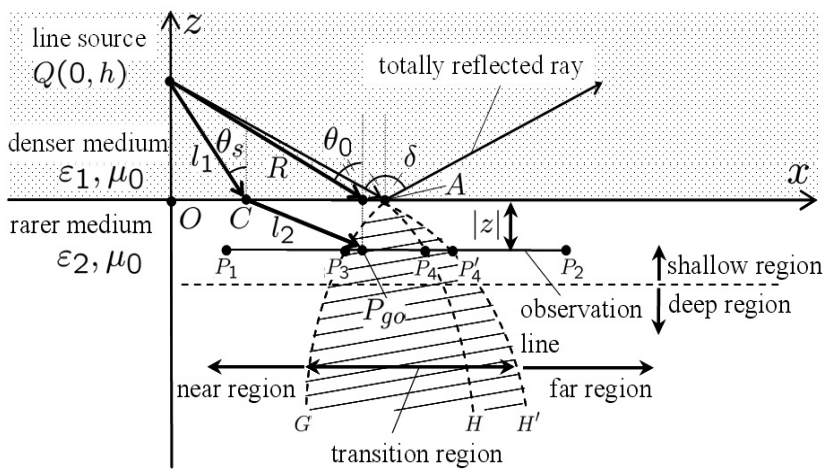

Fig. 4. Transmitted geometrical ray solution $G_{g o}$ observed at $P_{g o}$ located in the transition region between the dotted curves $A G$ and $A H$. 


$$
G=\frac{i}{4 \pi} \int_{P_{\theta_{S}}} T(\theta) e^{i k_{1} q(\theta)} d \theta
$$

Note that $G$ in (11) is the integral along the straight integration path $P_{\theta_{S}}$ (see Fig. 3). Since the steepest descent path $S D P_{\theta_{S}}$ in (6) is the curved integration path as shown in Fig. 3, it is difficult to derive from (6) the uniform asymptotic solution expressed by using the parabolic cylinder functions [24], [25]. If we deform the curved steepest descent path $S D P_{\theta_{S}}$ into the straight integration path $P_{\theta_{s}}$ as shown in Fig. 3, it becomes possible to derive the uniform asymptotic solution represented by using the parabolic cylinder functions as shown in the following. One may show that the integral in (6) is equal to the integral in (11) [19] [21], [23].

To proceed the asymptotic analysis of the integral $G$ in (11), the function $T(\theta)$ may be expressed as follows

$$
\begin{aligned}
T(\theta)= & T\left(\theta_{s}\right)+T^{d}(\theta) \\
& +T^{(2)}(\theta) \sqrt{\sin (\delta+\theta) \sin (\delta-\theta)} \\
T^{d}(\theta)= & T^{(1)}(\theta)-T^{(1)}\left(\theta_{\varsigma}\right) \\
& -T^{(2)}\left(\theta_{s}\right) \sqrt{\sin \left(\delta+\theta_{s}\right) \sin \left(\delta-\theta_{s}\right)} \\
T^{(1)}(\theta)= & \frac{2 \cos ^{2} \theta}{1-n^{2}}, T^{(2)}(\theta)=\frac{-2 \cos \theta}{1-n^{2}}
\end{aligned}
$$

Then the integral (11) may be represented by

$$
G=G_{1}+G_{2}+G_{3}
$$

where $G_{1}, G_{2}$, and $G_{3}$ can be represented by (11) with the replacement of $T(\theta)$ in (11) by $T\left(\theta_{S}\right), T^{d}(\theta)$, and $T^{(2)}(\theta) \sqrt{\sin (\delta+\theta) \sin (\delta-\theta)}$, respectively [20], [21], [23].

The integral $G_{1}$ for example may be represented by

$$
\begin{aligned}
G_{1}= & \frac{i}{4 \pi} T\left(\theta_{S}\right) e^{i k_{1} q\left(\theta_{s}\right)} \int_{P_{\theta_{S}}} e^{U(\theta)+V(\theta)} d \theta \\
U(\theta) & =i k_{1}|z|\left(\sqrt{n^{2}-\sin ^{2} \theta}-\sqrt{n^{2}-\sin ^{2} \theta_{s}}\right) \\
V(\theta) & \doteqdot i k_{1} R\left\{\left(\theta-\theta_{s}\right)^{2} \frac{\cos \left(\theta_{s}-\theta_{0}\right)}{2}\right. \\
& \left.+\left(\theta-\theta_{s}\right) \sin \left(\theta_{s}-\theta_{0}\right)\right\}
\end{aligned}
$$

When $U(\theta)$ in $e^{U(\theta)}$ in (16) satisfies the condition $|U(\theta)| \ll 1$, then one may substitute the expansion

$$
e^{U(\theta)}=\sum_{j=0}^{\infty} \frac{\{U(\theta)\}^{j}}{j !}
$$

into (16) and perform the lengthy but straightforward calculation to derive

$$
\begin{aligned}
& G_{1}=\frac{i}{4 \pi} T\left(\theta_{S}\right) e^{i k_{1} l_{1}+i k_{2} l_{2}-i \pi / 4} I_{1} \\
& I_{1}=\sum_{j=0}^{\infty} \frac{\alpha^{j}}{\sqrt{k_{1} \operatorname{Rcos}\left(\theta_{s}-\theta_{0}\right)} j !} I(j) \\
& I(j)=\int_{-\infty}^{\infty}(\sqrt{\beta+w}-\sqrt{\beta})^{j} e^{-\frac{w^{2}}{2}+\gamma w} d w
\end{aligned}
$$

where $\alpha, \beta$, and $\gamma$ are defined as follows.

$$
\begin{aligned}
& \alpha=i k_{1}|z| e^{-i \pi / 8} \frac{\sqrt{\sin \left(\delta+\theta_{s}\right) \cos \left(\delta-\theta_{s}\right)}}{\left\{k_{1} R \cos \left(\theta_{s}-\theta_{0}\right)\right\}^{1 / 4}} \\
& \beta=e^{i \pi / 4} \tan \left(\delta-\theta_{s}\right) \sqrt{k_{1} R \cos \left(\theta_{s}-\theta_{0}\right)} \\
& \gamma=e^{i \pi / 4} \frac{k_{1} R \sin \left(\theta_{0}-\theta_{s}\right)}{\sqrt{k_{1} \operatorname{Rcos}\left(\theta_{s}-\theta_{0}\right)}}
\end{aligned}
$$

The integral $I(j)$ in (22) is proceeded furthermore by applying the binominal theorem [29]

$$
\begin{array}{r}
(\sqrt{\beta+w}-\sqrt{\beta})^{j}=\sum_{m=0}^{j}{ }_{j} C_{m} \\
\cdot(\sqrt{\beta+w})^{m}(-\sqrt{\beta})^{j-m}
\end{array}
$$

where ${ }_{i} C_{m}$ denotes the binominal coefficient [29], and then transforming from the complex $w$-plane to the complex $s$-plane via $s=\beta+w$. The integral (22) may be represented by

$$
\begin{aligned}
I(j)= & e^{-\frac{\beta^{2}}{2}-\gamma \beta} \sum_{m=0}^{j}{ }_{j} C_{m}(-\sqrt{\beta})^{j-m} \\
& \cdot\left\{I_{m, 1}+I_{m, 2}\right\} \\
I_{m, 1}= & \int_{0}^{\infty} s^{m / 2} e^{-\frac{s^{2}}{2}-(\gamma-\beta) s} d s \\
I_{m, 2}= & i^{m} \int_{0}^{\infty} s^{m / 2} e^{-\frac{s^{2}}{2}+(\gamma-\beta) s} d s
\end{aligned}
$$

Above integrals $I_{m, 1}$ and $I_{m, 2}$ are expressed by using the parabolic cylinder functions [24], [25]. One may obtain the following result for $I(j)$.

$$
\begin{aligned}
I(j)= & \sum_{m=0}^{j} i^{m / 2} \sqrt{2 \pi}{ }_{j} C_{m}(-\sqrt{\beta})^{j-m} \\
& \cdot e^{\gamma^{2} / 2-(\gamma-\beta)^{2} / 4} D_{m / 2}(i\{\gamma-\beta\})
\end{aligned}
$$

In deriving (30), we have applied the relation for the parabolic cylinder function as follows [24], [25];

$$
D_{n}(z)=\frac{\Gamma(n+1)}{\sqrt{2 \pi}}\left\{e^{i \frac{n \pi}{2}} D_{-n-1}(i z)+e^{-i \frac{n \pi}{2}} D_{-n-1}(-i z)\right\}
$$


where $\Gamma(z)$ and $D_{v}(z)$ denote, respectively, the Gamma function and the parabolic cylinder function [24], [25].

Similarly, one may obtain the asymptotic solution for $\left(G_{2}+G_{3}\right)$ in (15) as follows;

$$
\begin{aligned}
G_{2}+G_{3}= & \frac{i}{4 \pi} T^{(2)}\left(\theta_{s}\right) \sqrt{\sin \left(\delta+\theta_{s}\right)} \\
& \cdot e^{i k_{1} l_{1}+i k_{2} l_{2}-\frac{i \pi}{4}}\left(I_{2}+I_{3}\right) \\
I_{2}+I_{3}= & \sum_{j=0}^{\infty} \frac{e^{-i \pi / 8} \sqrt{\cos \left(\delta-\theta_{s}\right)} \alpha^{j}}{\left\{k_{1} \operatorname{Rcos}\left(\theta_{s}-\theta_{0}\right)\right\}^{3 / 4} j !} I(j+1)
\end{aligned}
$$

Substituting $G_{1}$ in (20) associated with (30) and $G_{2}$ $+G_{3}$ in (32) associated with (33) into (15) produces the asymptotic solution for $G$ and then substituting $G$ thus obtained into (10) one may obtain the transition wave $G_{\text {tran }}$ which plays an important role in the transition region between the dotted curves $A G$ and $A H$ (see Figs. 1 and 4).

When the observation point moves along the observation line $P_{1} P_{2}$ to the $P_{1}$-direction (see Fig. 4), the transition wave $G_{\text {tran }}$ approaches zero, i.e., $G_{\text {tran }} \rightarrow 0$, outside the transition region where $x<P_{3}$ (see Fig. 4). Thus the transmitted wave $G$ in (7) in the near region is represented only by the transmitted geometrical ray $G_{g o}$. Since the asymptotic solution for (7) is applicable in the transition region and in the near region, we define the asymptotic solution for (7) as the uniform asymptotic solution. The uniform asymptotic solution for $G$ in (7) derived in this section is new and plays an important role in the transition region between the dotted curves $A G$ and $A H$ and approaches the transmitted geometrical ray $G_{g o}$ in the near region.

\section{3-2 Asymptotic Solution in Transition Region and in Far Region}

When the observation point is located in the transition region between the dotted curves $A H$ and $A H^{\prime}$ (see Fig. 1), two saddle points $\theta_{S}$ and $\theta_{e}$ are obtained from the saddle point equation (5). In Fig. 5, we have shown the steepest descent paths $S D P_{\theta_{S}}$ and $S D P_{\theta_{e}}$ passing through the saddle points $\theta_{S}$ and $\theta_{e}$, respectively.

The transmitted wave $G$ in (1) may be represented as [6], [20] [23]

$$
\begin{aligned}
& G=G_{e, v a}+\hat{G} \\
& G_{\text {eva }}=\frac{i}{4 \pi} \int_{S D P_{\theta_{e}}} T(\theta) e^{i k_{1} q(\theta)} d \theta \\
& \widehat{G}=\frac{i}{4 \pi} \int_{S D P_{\theta_{S}}} T(\theta) e^{i k_{1} q(\theta)} d \theta
\end{aligned}
$$

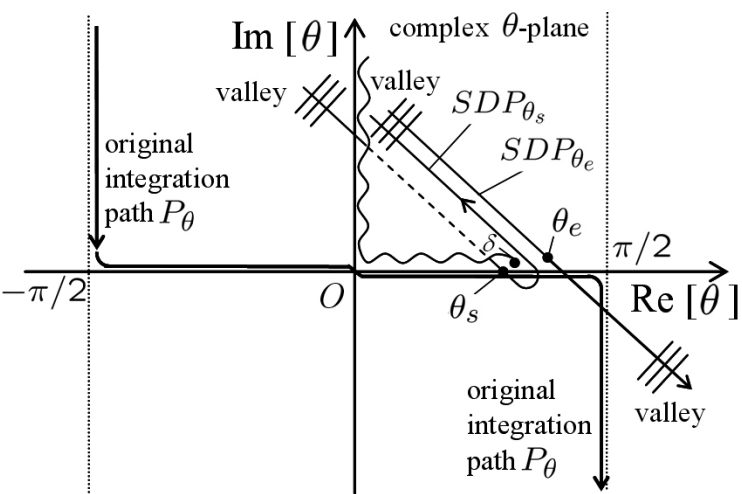

Fig. 5. Steepest descent paths $S D P_{\theta_{S}}$ and $S D P_{\theta_{e}}$ passing through saddle points $\theta_{S}$ and $\theta_{e}$, respectively, for observation point placed in the transition region and in the far region.

Since the saddle point $\theta_{e}$ is located away from $\theta_{s}$ and the branch point at $\theta=\delta$ (see Fig. 5), the integral $G_{\text {eva }}$ representing the evanescent wave is evaluated by applying the isolated saddle point technique [28]. The asymptotic solution for $G_{\text {eva }}$ may be represented as

$$
\begin{aligned}
& G_{e v a} \sim \frac{i}{4 \pi} \sqrt{\frac{2}{\pi k_{1} R}} e^{i k_{1} R-i \frac{\pi}{4}} T\left(\theta_{0}\right) \\
& \cdot e^{-k_{1}|z| \sqrt{\sin ^{2} \theta_{0}-n^{2}}}
\end{aligned}
$$

where $\theta_{0} \doteqdot \operatorname{Re}\left[\theta_{e}\right]$. The evanescent wave $G_{\text {eva }}$, excited by the incident wave $Q \rightarrow E$ (see Fig. 1) with the incident angle $\theta_{0}$ satisfying $\theta_{0}>\delta$ (critical angle of the total reflection), decays exponentially rapidly in the $-z$ direction as shown in Fig. 1.

\section{3-2-1 Asymptotic Solution in Deep Region}

When the observation point is located in the transition region between $A H$ and $A H^{\prime}$ or in the far region in the deep region (see Fig. 1), by applying the isolated point technique [28] one may obtain from $\hat{G}$ in (36) the transmitted geometrically ray solution $G_{g o}, Q(0, h) \rightarrow D \rightarrow P_{2}$ shown in Fig. 1 (Note that the observation point $P_{2}$ is placed in the deep region in this case). The transmitted geometrical ray $G_{g o}$ derived from (36) agrees with the solution $G_{g o}$ derived in (8) and (9) for the transmitted geometrical ray $Q(0, h) \rightarrow C \rightarrow P_{g o}$.

Thus the asymptotic solution in the transition region and in the far region in the deep region may be obtained from the summation of the evanescent wave $G_{\text {eva }}$ $\left(Q(0, h) \rightarrow E \rightarrow P_{2}\right)$ and the transmitted geometrical ray $G_{g o}\left(Q(0, h) \rightarrow D \rightarrow P_{2}\right)$ (see Fig. 1). However, in the deep region, the evanescent $G_{\text {eva }}$ in (37) is sufficiently 
small so that the transmitted wave $G$ in (34) may be obtained only from the transmitted geometrical ray solution as follows.

$$
G \sim G_{g o}
$$

\section{3-2-2 Asymptotic Solution in Shallow Region}

In this section, we shall derive from (34) (36) the uniform asymptotic solution for the observation point placed in the transition region between the curves $A H$ and $A H^{\prime}$ and in the far region in the shallow region (see Fig. 1). In this case, the saddle point $\theta_{S}$ is located near the branch point at $\theta=\delta$ (see Fig. 5). The asymptotic solution for the evanescent wave $G_{e v a}$ in (37) is unchanged.

To derive the asymptotic solution for $\widehat{G}$ in (36), one may express the function $T(\theta)$ in (36) as expressed in (12) associated with (13) and (14). Then the integral $\hat{G}$ may be represented by

$$
\widehat{G}=\widehat{G}_{1}+\widehat{G}_{\eta}+\widehat{G}_{3}
$$

where $\widehat{G}_{1}, \widehat{G}_{7}$, and $\hat{G}_{3}$ are given by (36) with the replacement of $T(\theta)$ in (36) by $T\left(\theta_{s}\right), T^{d}(\theta)$, and $T^{(2)}(\theta) \sqrt{\sin (\delta+\theta) \sin (\delta-\theta)}$, respectively (see (12)) [6], [20] [23].

The integral $\hat{G}_{3}$ for example may be approximated by

$$
\begin{aligned}
& \widehat{G}_{3} \doteqdot \frac{i}{4 \pi} T^{(2)}\left(\theta_{s}\right) \sqrt{\sin \left(\delta+\theta_{s}\right)} e^{i k_{1} q\left(\theta_{s}\right)} I_{3} \\
& I_{3}=\int_{S D P_{\theta_{S}}} \sqrt{\sin (\delta-\theta)} e^{U(\theta)+V(\theta)} d \theta
\end{aligned}
$$

where the functions $U(\theta)$ and $V(\theta)$ have been defined in (17) and (18), respectively.

Note that the integration path $P_{\theta_{S}}$ for $G$ in (11) and $G_{1}, G_{2}$, and $G_{3}$ in (15) (see Fig. 3) is different from the steepest descent path $S D P_{\theta_{S}}$ for $\widehat{G}$ in (36) and $\widehat{G}_{1}, \hat{G}_{\eta}$, and $\widehat{G}_{3}$ in (39). After passing through the saddle point $\theta_{s}$, the $S D P_{\theta_{S}}$ turns $360^{\circ}$ around the branch point at $\theta=\delta$ in the counterclockwise direction and runs along the straight line to the backward direction toward the valley located in the upper left region in the 1st quadrant of the complex $\theta$-plane as shown in Fig. 5. Thus, the analysis method applied in the followings is different from the one applied in the Section 3-1.

Assuming $|U(\theta)| \ll 1$, the function $e^{U(\theta)}$ in (41) is expanded as in (19). Then the integral $I_{3}$ in (41) is evaluated along the steepest descent path $S D P_{\theta_{S}}$ shown in
Fig. 5. In the papers [6] and [23], we have derived the asymptotic solution for the reduced integral $I_{3}\left(=I_{3}^{-}\right.$ $+I_{3}^{+}$). The integrals $I_{2}^{\mp}$ derived in [20] [22] have been solved by using the parabolic cylinder functions [6], [23] between the dotted curves $A H$ and $A H^{\prime}$ for the saddle point $\theta_{S}$ located near the branch point $\delta$, i.e., $\theta_{S} \doteqdot \delta$. By performing the straightforward calculations similar to those applied for $I_{2}^{\mp}$, we have also derived in [6] and [23] the asymptotic solution for the integrals $\hat{G}_{1}$ and $\hat{G}_{2}$ by using the parabolic cylinder functions [6], [23] [25].

Thus we will obtain the novel uniform asymptotic solution for $G$ in (34) as follows

$$
G=G_{\text {eva }}+G_{\text {tran }}+G_{\text {lat.wave }}
$$

where $G_{\text {tran }}\left(=\widehat{G}_{1}+\widehat{G}_{2}\right)$ denotes the transition wave solution which plays the important roles only in the transition region [6], [20] [23] (see Fig. 1) and $G_{\text {lat.wave }}$ $\left(=\widehat{G}_{3}\right)$ denotes the lateral wave type transmitted wave. $\hat{G}_{1}, \hat{G}_{7}$, and $\hat{G}_{3}$ may be represented by [6], [23]

$$
\begin{aligned}
& \widehat{G}_{1} \sim \frac{i}{4 \pi} T\left(\theta_{S}\right) e^{i k_{1} L_{1}+i k_{2} L_{2}-i \pi / 4}\left(I_{1}^{-}+I_{1}^{+}\right) \\
& I_{1}^{\mp} \sim \pm \sum_{j=0}^{\infty} \frac{1}{\sqrt{k_{1} R \cos \left(\theta_{s}-\theta_{0}\right)}} \frac{\left(\alpha^{\mp}\right)^{j}}{j !}
\end{aligned}
$$

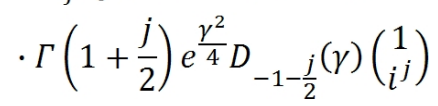

$$
\begin{aligned}
& \widehat{G}_{2} \sim-\frac{i}{4 \pi} T^{(2)}\left(\theta_{s}\right) \sqrt{\sin \left(\delta+\theta_{s}\right) \sin \left(\delta-\theta_{s}\right)} \\
& \cdot e^{i k_{1} L_{1}+i k_{2} L_{2}-i \pi / 4}\left(I_{?}^{-}+I_{2}^{+}\right), I_{2}^{\mp}=I_{1}^{\mp} \\
& \widehat{G}_{3} \sim-\frac{i}{4 \pi} T^{(2)}\left(\theta_{s}\right) \sqrt{\sin \left(\delta+\theta_{s}\right)} \\
& \cdot e^{i k_{1} L_{1}+i k_{2} L_{2}-i \pi / 4}\left(I_{3}^{-}+I_{3}^{+}\right) \\
& I_{3}^{\mp} \sim \pm \sum_{j=0}^{\infty} \frac{\sqrt{\cos \left(\delta-\theta_{s}\right)}}{\left\{k_{1} \operatorname{Rcos}\left(\theta_{s}-\theta_{0}\right)\right\}^{3 / 4}} \frac{\left(\alpha^{\mp}\right)^{j}}{j !} \\
& \cdot \Gamma\left(\frac{j+3}{2}\right) e^{\frac{\gamma^{2}}{4}} D_{-\frac{j+3}{2}}(\gamma)\left(\begin{array}{l}
e^{-i \pi / 8} \\
e^{i 3 \pi / 8}
\end{array}\right)
\end{aligned}
$$

where $\alpha^{\mp}$ and $\gamma$ are defined as follows.

$$
\begin{aligned}
& \alpha^{\mp}=i k_{1}|z| \frac{\sqrt{\sin \left(\delta+\theta_{s}\right) \cos \left(\delta-\theta_{s}\right)}}{\left\{k_{1} \operatorname{R} \cos \left(\theta_{s}-\theta_{0}\right)\right\}^{1 / 4}}\left(\begin{array}{c}
e^{-i \pi / 8} \\
e^{i 3 \pi / 8}
\end{array}\right) \\
& \gamma=e^{i \pi / 4} \frac{k_{1} R \sin \left(\theta_{0}-\theta_{s}\right)}{\sqrt{k_{1} R \cos \left(\theta_{s}-\theta_{0}\right)}}
\end{aligned}
$$

Note that $I_{1}^{+}$in (44), for example, chooses the lower signs. That is - sign from,$\pm \alpha^{+}$from $\alpha^{\mp}$, and $i^{j}$ from 
$\left(1\right.$ and $\left.i^{j}\right)$ in the open brackets ( ).

As far as authors know, (42) is the new solution applicable in the transition region between the dotted curves $A H$ and $A H^{\prime}$ and in the far region.

When the observation point $P_{2}$ is located in the shallow and far region, the novel uniform asymptotic solution in (42) may be approximated by

$$
G \sim G_{\text {eva }}+G_{\text {lat. wave }}^{0}
$$

where $G_{\text {lat.wave }}^{0}$, obtained from (46) and (47) by substituting $j=0$ and the asymptotic approximation for the parabolic cylinder function $D_{-3 / 2}(\gamma)$ [24], [25], is given by [6], [20], [22], [23]

$$
G_{\text {lat.wave }}^{0}=\frac{i}{k_{1}} \sqrt{\frac{2 n}{\pi k_{1}}} \frac{e^{i 3 \pi / 4}}{1-n^{2}} \frac{1}{L_{2}^{3 / 2}} e^{i k_{1} L_{1}+i k_{2} L_{2}}
$$

The geometrical distances $L_{1}$ and $L_{2}$ in (51) are defined in Fig. 1. It is very interesting to observe that in the shallow and far region the amplitude $1 /\left(L_{2}^{3 / 2}\right)$ of $G_{\text {lat.wave }}^{0}$ in (51) behaves like a lateral wave which is usually observed in the denser 1st medium (see Fig. 1). Thus we define the transmitted wave $G_{\text {lat.wave }}^{0}$ in (51) as the lateral wave type transmitted wave. While $G_{e v a}$ in (50) is the evanescent wave derived in (37).

\section{Numerical Results and Discussions}

In this section, we will confirm the validity and applicability of the novel uniform asymptotic solutions in (7) and (42) and the asymptotic solution in (50) numerically. We have performed extensive numerical calculations to show the validity of the novel uniform asymptotic solution presented in this paper. However, we will present here three typical examples since the results calculated by using other numerical parameters are similar to those shown here.

In Fig. 6, we have shown the electric field magnitude vs. distance $x(\lambda)$ curve. We have calculated the transmitted wave observed in the rarer medium when the observation point is located in deep region $z=-2.5 \lambda$. The bold solid curve (-) calculated by using the transmitted geometrical rays $G_{g o}$ in (8) and (38) agrees excellently with the reference solution $(\bullet \bullet \bullet$ : closed circles) calculated numerically from (1). It is clarified that when the observation point is located in the deep region, only the transmitted geometrical ray provides the excellent solution.

For the observation point located in the shallow region, the typical numerical result is shown in Fig. 7 for the case when $z=-0.7 \lambda$. The numerical parameters

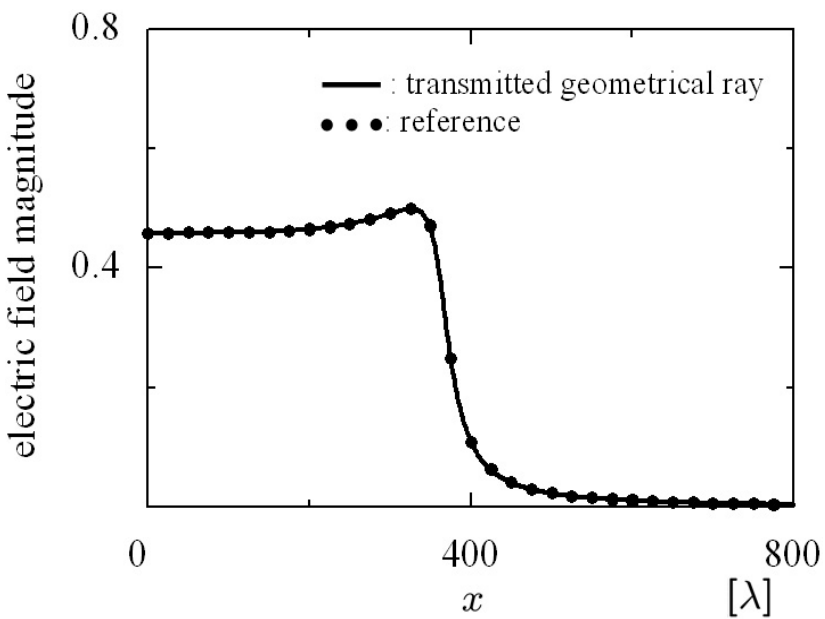

Fig. 6. Comparison of transmitted geometrical ray solution with the reference solution at the depth $z=$ $-2.5 \lambda$. Numerical parameters: $h=400 \lambda, \varepsilon_{1}=2.3 \varepsilon_{0}$, $\varepsilon_{2}=1.0 \varepsilon_{0}, f=3 \mathrm{GHz}, n=0.659$.

used in the calculation are the same as those used in the calculation of Fig. 6. The thin solid curve (-) is calculated by using the uniform asymptotic solution in (7) in the near region and in the transition region between the curves $A G$ and $A H$ and the uniform asymptotic solution in (42) in the transition region between the curves $A H$ and $A H^{\prime}$ and in the far region (see Figs. 1 and 4). It is clarified that the uniform asymptotic solution (-) derived in this study agrees very well with the reference

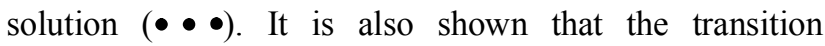
waves (-'-) calculated from the solution $G_{\text {tran }}$ in (10) and $G_{\text {tran }}=\widehat{G}_{1}+\widehat{G}_{\text {? }}$ (see (42)) play an important role in the transition region $P_{3}<x<P^{\prime}{ }_{4}$ shown in Figs. 1 and 4 .

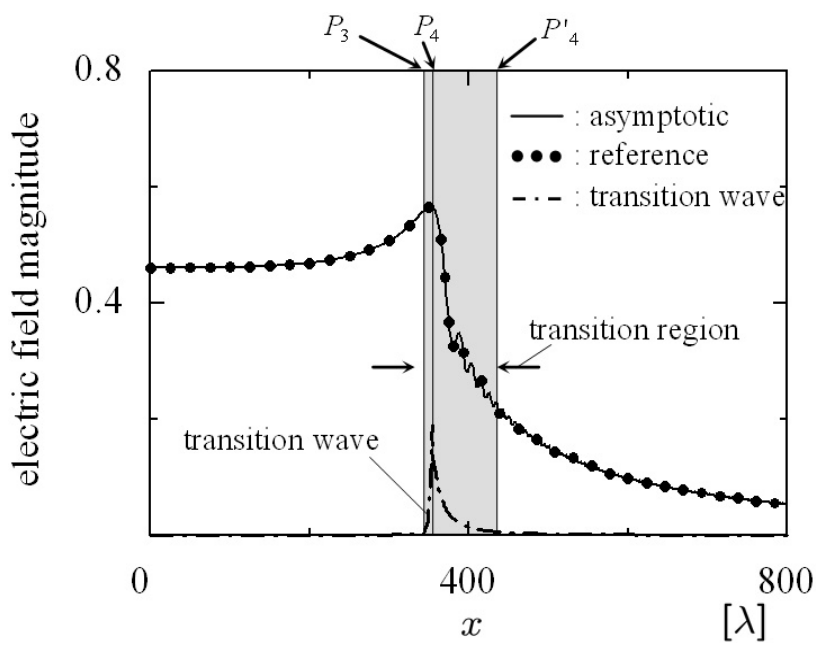

Fig. 7. Comparison of uniform asymptotic solution with reference solution at the depth $z=-0.7 \lambda$. 


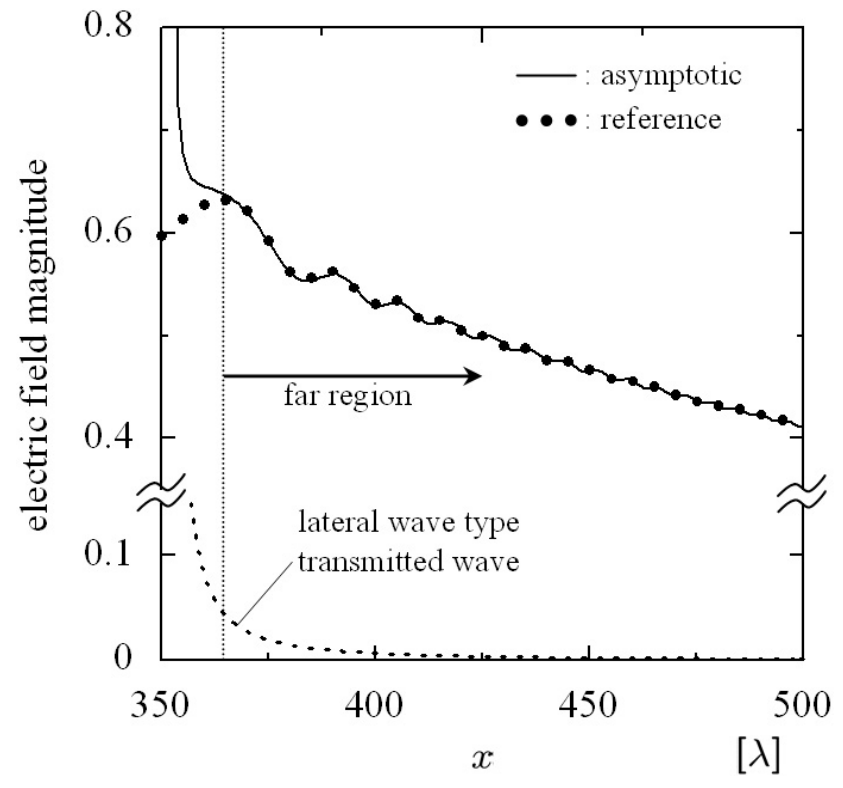

Fig. 8. Comparison of asymptotic solution in (50) with the reference solution. Numerical parameters: $h=$ $400 \lambda, \quad \varepsilon_{1}=2.3 \varepsilon_{0}, \quad \varepsilon_{2}=1.0 \varepsilon_{0}, f=3 \mathrm{GHz}$, and $z=-0.05 \lambda$ (shallow region).

In Fig. 8, we have calculated the transmitted wave $G$ in the shallow $(z=-0.05 \lambda)$ and far region by using the asymptotic solution (50) consisting of the evanescent wave in (37) and the lateral wave type transmitted wave in (51). It is clarified that, the asymptotic solution (-) agrees excellently with the reference solution $(\bullet \bullet \bullet$ : closed circles) calculated numerically from (1) in the far region shown in Fig. 8. However, the asymptotic solution deviates from the reference solution in the region where $x<365 \lambda$ (see Fig. 8) since the approximations we have applied become inaccurate in this region. Thus we have confirmed that the lateral wave type transmitted wave in (51) is valid in the far and shallow region. In Fig. 8, we have also plotted the lateral wave type transmitted wave in (51) by the dotted curve ( ……). By the interference of the evanescent wave and the lateral wave type transmitted wave, the asymptotic solution oscillates as the function of the horizontal distance $x(\lambda)$.

It is very interesting to observe that the lateral wave type transmitted wave with the amplitude proportional to $1 /\left(L_{2}^{3 / 2}\right)$ (see Fig. 1) is excited near the plane dielectric interface where $|z|$ is small.

\section{Conclusions}

We have derived the novel uniform asymptotic solution represented by using the parabolic cylinder function for the transmitted wave observed in the rarer medium when the cylindrical wave is incident on a plane dielectric interface from the denser medium. We have confirmed the validity and utility of the uniform asymptotic solution by comparing with the reference solution calculated numerically. It is clarified that the transition wave plays an important role to connect smoothly the two different solutions in the near region and the far region through the transition region. We have shown the very interesting phenomenon that the lateral wave type transmitted wave is produced in the shallow and far region.

This work was supported in part by the Grant-in-Aid for Scientific Research (C) (21560424) from Japan Society for the Promotion of Science (JSPS).

\section{References}

[1] M. Hashimoto, "The law of total reflection in geometrical optics based on wave-normal rays," IEICE Trans. Electron. C-I, vol. J72-C-I, no. 3, pp. 131138, Mar. 1989.

[2] H. Shirai, "Transient scattering responses from a plane interface between dielectric half space," IEICE Trans. Electron. C-I, vol. J78-C-I, no. 3, pp. 125133, Mar. 1995.

[3] H. Yamada, T. Kawano, K. Goto, and T. Ishihara, "A uniform asymptotic solution for lateral displacement of a Gaussian beam at a dielectric interface," Proc. of International Symposium on Antennas and Propagation (ISAP) 2007, CD-ROM (ISBN: 978-488552-223-9C3055@IEICE), Niigata, Japan, Aug. 2007.

[4] D. T. Quang, K. Goto, T. Kawano, and T. Ishihara, "A novel uniform asymptotic solution for reflection of a Gaussian beam at a dielectric interface," IEICE Electron. Express, vol. 8, no. 6, pp. 397-403, Mar. 2011.

[5] D. T. Quang, K. Goto, T. Kawano, and T. Ishihara, "A uniform asymptotic solution for reflection and beam shift of a Gaussian beam at a plane dielectric interface," IEICE Trans. Electron., vol. E95-C, no. 1, pp. 16-26, Jan. 2012.

[6] D. T. Quang, K. Goto, T. Kawano, and T. Ishihara, "A novel uniform asymptotic solution for transmitted wave through a plane dielectric interface," IEICE Electron. Express, vol. 8, no. 23, pp. 1989-1995, Dec. 2011.

[7] H. L. Bertoni, L. B. Felsen, and J. W. Ra, "Evanescent fields produced by totally reflected beams," IEEE Trans. on Antennas and Propag., pp. 730-732, Sep. 1973.

[8] Q. Li, R. J. Vernon, "Theoretical and experimental 
investigation of Gaussian beam transmission and reflection by a dielectric slab at $110 \mathrm{GHz}$," IEEE $\mathrm{Tr}$ ans. Antennas Propag., vol. 54, no. 11, pp. 34493457, Nov. 2006.

[9] H. K. V. Lotsch, "Beam displacement at total reflection: The Goos-Hänchen effect I," Optik, vol. 32, no. 2, pp. 116-137, Apr. 1970.

[10] B. R. Horowitz, T. Tamir, "Lateral displacement of light beam at a dielectric interface," J. Opt. Soc. Am., vol. 61, no. 5, pp. 586-594, May 1971.

[11] B. R. Horowitz, T. Tamir, "Unified theory of total reflection phenomena at a dielectric interface," Appl. Phys., 1, pp. 31-38, vol. 1, 1973.

[12] S. Kozaki, H. Sakurai, "Characteristics of a Gaussian beam at a dielectric interface," J. Opt Soc. Am., vol. 68, no. 4, pp. 508-514, Apr. 1978.

[13] L. M. Brekhovskikh, Waves in Layered Media, 2nd ed., chaps. 1 and 4, Academic Press, New York, USA, 1980.

[14] E. K. Westwood, "Complex ray methods for acoustic interaction at a fluid-fluid interface," J. Acoust. Soc. Am., 85(5), pp. 1872-1884, May 1989.

[15] D. R. Smith, D. Schurig, and J. B. Pendry, "Negative refraction of modulated electromagnetic waves," Applied Physics Letters, vol. 81, no. 15, pp. 2713-2715, Oct. 2002.

[16] P. M. Valanju, R. M. Walser, and A. P. Valanju, "Wave refraction in negative-index media: always positive and very inhomogeneous," Physical Review Letters, vol. 88, no. 18, pp. 187401-1-187401-4, May 2002.

[17] J. R. Thomas, A. Ishimaru, "Wave packet on negative index media," IEEE Trans. on Antennas and Propag., vol. 53, no. 5, pp. 1591-1599, May 2005.

[18] T. Kawano, K. Goto, and T. Ishihara, "High-frequency asymptotic analysis for scattered fields over half-space metamaterials," The Paper of Technical Meeting on Electromagnetic Theory, IEE Japan, EMT-10-131, pp. 165-170, Nov. 2010.

[19] D. T. Quang, K. Goto, T. Kawano, and T. Ishihara, "Asymptotic analysis of transmitted wave in transition region through a plane dielectric interface," The Paper of Technical Meeting on Electromagnetic Theory, IEE Japan, EMT-10-129, pp. 153-157,
Nov. 2010.

[20] D. T. Quang, K. Goto, T. Kawano, and T. Ishihara, "A novel asymptotic analysis for transmitted waves through a plane dielectric interface," The Paper of Technical Meeting on Electromagnetic Theory, IEE Japan, EMT-11-055, pp. 57-62, May 2011.

[21] D. T. Quang, K. Goto, T. Kawano, and T. Ishihara, "Novel asymptotic solution in the transition region for transmitted wave through a plane dielectric interface," IEEE AP-S Int. Symp., CD-ROM (ISBN: 978-1-4244-9561-0), Spokane, USA, Jul. 2011.

[22] D. T. Quang, K. Goto, T. Kawano, and T. Ishihara, "A uniform asymptotic solution for transmitted waves through a plane dielectric interface from a denser to a rarer mediums," Proc. of International Symposium on Antennas and Propagation (ISAP) 2011, ISBN: 978-89-5708-211-9 Jeju, Korea, Oct. 2011.

[23] D. T. Quang, K. Goto, T. Kawano, and T. Ishihara, "Asymptotic analysis of transmitted waves through a plane dielectric interface by using parabolic cylinder function," The Paper of Technical Meeting on Electromagnetic Theory, IEE Japan, EMT-11-130, pp. 13-19, Nov. 2011.

[24] E. T. Whittaker, G. N. Watson, A Course in Modern Analysis, 4th eds., pp. 347-351, Cambridge University Press, Cambridge, 1927.

[25] M. Abramowitz, I. A. Stegun, Eds., Handbook of Mathematical Functions, pp. 686-720, Dover, New York, 1972.

[26] J. P. Tignol, Galois' Theory of Algebraic Equations, chap. 3, World Scientific Publishing, Toh Tuck Link, Singapore, 2001.

[27] W. H. Press, B. P. Flannery, S. A. Teukolsky, and W. T. Vetterling, Numerical Recipes in FORTRAN: The Art of Scientific Computing, 2nd Ed., sec. 9.5, Cambridge University Press, Cambridge, England, 1992.

[28] L. B. Felsen, N. Marcuvitz, eds., Radiation and Scattering of Waves, chap. 4, IEEE Press (Classic Reissue), New Jersey, USA, 1994.

[29] M. Abramowitz, I. A. Stegun, Eds., Handbook of Mathematical Functions, p. 10, Dover, New York, 1972. 


\section{Dinh T. Quang}

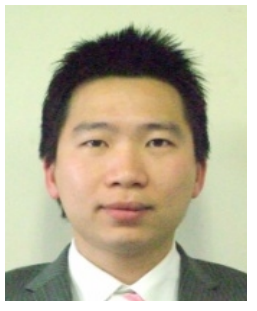

was born in Hanoi, Vietnam, in 1983. He received the B.S. and the M.S. degrees from Department of Electrical Engineering, National Defense Academy of Japan, Yokosuka, Japan, in 2008 and 2010, respectively. His current research interests are scattering, radiation, and propagation of electromagnetic waves. He received the Student Award from IEICE Electronics Society of Japan in 2009 and the Student Paper Presentation Award from Institute of Electrical Engineering of Japan in 2010.

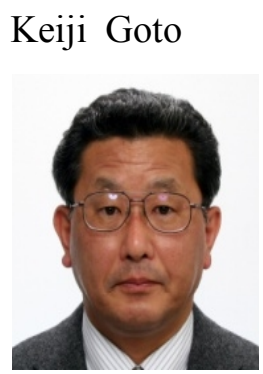

was born in Oita, Japan, in 1961. He received the B.S. and the M.S. degrees (equivalents) from Department of Electrical Engineering, National Defense Academy of Japan, Yokosuka, Japan, in 1984 an 1989, respectively, and the D.E. degree from the Tsukuba University, Tsukuba, Japan, in 2001. From 1991 to 1996, he was a Researcher at the National Defense Academy of Japan. From 1996 to 2004, he worked as a Researcher at the Japan Ground Self-Defense Forces and at the Defense Agency (now Ministry of Defense) in Japan. He was a Lecturer from 2004 to 2010 and is currently an Associate Professor with the Department of Communication Engineering, National Defense Academy of Japan. His research interests include high-frequency asymptotic analysis techniques of radiation and scattering of electromagnetic waves, such as uniform geometrical theory of diffraction and uniform physical optics. Dr. Goto received the Paper Presentation Award from Institute of Electrical Engineering of Japan in 1993.

\section{Toru Kawano}

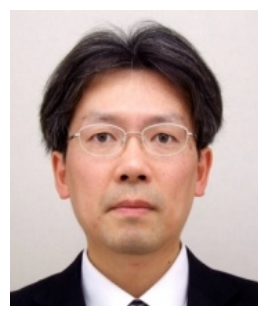

was born in Oita, Japan, on December 26, 1971. He received the B.E., M.E., and Dr. E. degrees in electrical engineering from Hosei University, Tokyo, Japan, in 1995, 1997, and 2001, respectively. From 2001 to 2010, he was an assistant with the National Defense Academy of Japan, Kanagawa, Japan. He is currently a Lecturer at the National Defense Academy. His current research interests are scattering, radiation, and propagation of electromagnetic waves. Dr. Kawano received the Paper Presentation Award from Institute of Electrical Engineering of Japan in 2008.

\section{Toyohiko Ishihara}

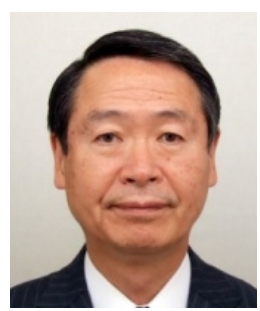

was born in Kumamoto, Japan, on January 21,1947 . He received the B.S. degree (equivalent) from the National Defense Academy of Japan, Yokosuka, Japan, and M.S. and Ph.D. degrees from the Polytechnic Institute of New York (now Polytechnic University), Brooklyn, NY, in 1970, 1975, and 1978, respectively. He received the Dr. of Eng. degree from Tohoku University, Sendai, Japan, in 1983. From 1979 to 1982, he was a Research Scientist at the Institute of Research and Development of Defense Agency in Japan. In 1982, he became a Lecturer at the National Defense Academy. From September 1984 through February 1985, he was a Visiting Scholar at the Polytechnic Institute of New York, Brooklyn, NY. He was an Associate Professor from 1985 to 1990 and is currently a Professor with the National Defense Academy. From 2007 to 2008, he was Chairman of the Research Committee on Electromagnetic Theory of the Institute of Electronics, Information and Communication Engineers (IEICE) in Japan. He served as Chairman of the 2000 Marine Acoustic Society of Japan Meeting. His research interests include highfrequency asymptotic analysis of radiation and scattering of electromagnetic waves, radio wave propagation, and acoustic wave propagation in underwater. Dr. Ishihara received the Defense Technology Journal Paper Award of the Defense Technology Foundation of Japan in 1985, the Paper Award of the Marine Acoustic Society of Japan in 1997, and the Sato Paper Award of the Acoustic Society of Japan in 1999. 